\title{
Genetic background of Citrus nobilis Lour. 'Gonggan' based on the chloroplast trnL gene
}

\author{
Y.-J. Guo ${ }^{1}$, Q.-H. Ji ${ }^{1}$, X.-Q. Zhou ${ }^{1}$, Y.-P. Hu ${ }^{1}$, L.-Y. Guo ${ }^{1}$, H. Jiang ${ }^{1}$ and \\ J.-W. Zeng ${ }^{2}$ \\ ${ }^{1}$ Fruit Tree Research Institute, Zhaoqing University, Guangdong, China \\ ${ }^{2}$ Fruit Tree Research Institute, \\ Guangdong Province Academy of Agricultural Sciences, Guangdong, China \\ Corresponding author: Q.-H. Ji \\ E-mail: qhgee@hotmail.com
}

Genet. Mol. Res. 12 (3): 3079-3087 (2013)

Received April 16, 2012

Accepted October 15, 2012

Published January 30, 2013

DOI http://dx.doi.org/10.4238/2013.January.30.7

\begin{abstract}
The genetic backgrounds of many Citrus varieties are quite complex. Classifications and phylogenetic relationships of Citrus species have become the focus of researchs. Some conserved genes of chloroplast genome's research have been proven effective in determining the biosources of hybrids and phylogenetic analysis. Thus, we studied variations among the chloroplast trnL gene sequences of 10 Citrus species, including C. nobilis Lour. 'Gonggan'. The amplification results of different trnL target genes and identification of the double-enzyme cut after cloning show that lengths of all trnL sequences were within 895 to $935 \mathrm{bp}$ and a total of 24 variation sites were detected among the 10 material samples. Clustering analysis revealed differences in trnL genes caused by systematic evolution and allowed the determination of variations among Citrus varieties. Variation sites of trnL sequences can be used in the phylogenetic classification and species identification of Citrus, and the results agreed with
\end{abstract}


random amplified polymorphic DNA marker results. C. nobilis Lour. 'Gonggan' is closely associated with the other two varieties in Zhaoqing area, and C. nobilis Lour. 'Gonggan' and C. haniana Hort. ex Tseng 'Sihuihanggan' can be classified into the same category. C. nobilis Lour. 'Gonggan' as a natural hybrid is probably a hybrid with $C$. haniana Hort. ex Tseng 'Sihuihanggan' as its female parent.

Key words: Citrus nobilis Lour. 'Gonggan'; trnL; Citrus; Genetic background; Genetic relationship

\section{INTRODUCTION}

The classifications and phylogenetic relationships of Citrus species have been debated within academic circles ever since Citrus was defined in 1753 by the Swedish scientist Carolus Linnaeus. Thus far, systematic research on the classifications and genetic origins of Citrus plants has consisted of morphological and cytological studies, numerical classification, molecular markers, and so on, with many foreign and domestic scholars achieving a certain extent of progress (Xie et al., 2008; Ji and Guo, 2011). Several analytical methods for studying the systematic evolutions of conserved genes (e.g., rbcL) have been proven effective in determining the biosources of hybrids because chloroplast genomes are inherited from the female parent (Green et al., 1986; Wang and Jiang, 2010).

Citrus can easily undergo cross-breeding and open pollination, so natural hybrid varieties such as C. nobilis Lour. 'Gonggan' exist everywhere. Thus, the genetic backgrounds of many Citrus varieties are quite complex. While the morphological characteristics of some Citrus varieties are distinctly different, their genetic relationships may be very closely related. Studies on the chloroplast genomes of Citrus varieties can provide solid evidence regarding their genetic structures and classification statuses. In addition, they can also generate meaningful guidance for further cultivation of improved varieties.

C. nobilis Lour. 'Gonggan' has an orange-like shape and a thin golden peel. It is fragrant, tastes like honey, has an exterior similar to oranges, and is easy to peel like tangerines. C. nobilis Lour. 'Gonggan' is a highly productive and local characteristic species in farm lands of the ZhaoQing region. It was chosen as a tribute fruit for the imperial family during the Ming and Qing Dynasties because of its excellent quality. It is currently the second most-cultivated fruit in the ZhaoQing region, with sugar tangerines being the first. Deqing County in the ZhaoQing region is one of the main cultivation sites of C. nobilis Lour. 'Gonggan'. According to the Sihui County Record from the Qing Dynasty, the fruit originated from the Sihui region of ZhaoQing and is a natural hybrid of orange and tangerine. However, the species of its parents can no longer be determined. The Sihui region historically possesses rich Citrus resources; over 20 cultivars or more than 30 species of Citrus have been planted in the Sihui region. C. nobilis Lour. 'Gonggan' is believed to have relationships with several historical plant species (Ji et al., 2010).

The present study determines variations among the trnL gene sequences of 10 Citrus species, including C. nobilis Lour. 'Gonggan', to provide molecular evidence of the genetic background of Citrus and the origin of C. nobilis Lour. 'Gonggan'. 


\section{MATERIAL AND METHODS}

Ten test material samples were prepared. Samples included C. nobilis Lour. 'Gonggan', C. haniana Hort. ex Tseng 'Sihuihanggan', Citrus flamea Hort. ex Tseng 'Shiyueju', Citrus sinensis Osbeck 'Lanhuacheng', Atalantia buxifolia (Poir.) Oliv., Citrus grandis (L.) Osbeck 'Guanximiyou', Poncirus trifoliata (L.) Raf, Citrus limon (L.) Burm. f. 'Youlikeningmeng' (Eureka lemon), Citrus sinensis Osbeck 'Niuheerqicheng' (Newhall navel orange), and Citrus nobilis Lour. 'Xingjinwenzhoumigan' (Okitsu wase). The suspected parent of $C$. nobilis Lour. 'Gonggan' (C. haniana Hort. ex Tseng 'Sihuihanggan') is the dominant species currently cultivated in the Zhaoqing region (C. nobilis Lour. 'Gonggan' and C. flamea Hort. ex Tseng 'Shiyueju').

DNA extraction was performed as follows: 1) young and healthy Citrus lamina were selected; 2) genomes were extracted using the modified hexadecyltrimethylammonium bromide (CTAB) method ( $\mathrm{Ji}$ and Guo, 2011); 3) DNA purity and concentration were measured by $1 \%$ agarose gel electrophoresis and a micro-spectrophotometer; 4) $100 \mathrm{ng}$ DNA was obtained according to the DNA concentration to be used as polymerase chain reaction (PCR) template.

Amplification and clone and sequence analyses of trnL target bands utilized the following trnL amplification primers: TrnlP5: 5'-TAAAAATGGGTAATCCTGAGCCAA$3^{\prime}$ and TrnlP3: 5'-CCAGCTGAGCTACCCGAC-3'. The amplification protocol was $94^{\circ} \mathrm{C}$ for $3 \mathrm{~min}$; 40 cycles of $94^{\circ} \mathrm{C}$ for $30 \mathrm{~s}, 60^{\circ} \mathrm{C}$ for $30 \mathrm{~s}$, and $72^{\circ} \mathrm{C}$ for $1 \mathrm{~min}$; and a final extension at $72^{\circ} \mathrm{C}$ for $10 \mathrm{~min}$.

The PCR outcome was tested by $1.5 \%$ agarose gel electrophoresis. Some of the gel slices with special target bands displayed in the electrophoretogram were recovered and submitted to T-A cloning. The PCR outcome was connected to the T Carrier (pMD19T, Takara). Recombinants were continuously transformed into the Top 10 competent cells. Cells were revived at $37^{\circ} \mathrm{C}$ for $40 \mathrm{~min}$ and spread on a plate (Luria-Bertani medium with ampicillin). The plate was placed upside down overnight at a constant temperature for cultivation. PCR amplification was performed using the DNA of single-colony plasmids as template. The amplification protocol was as follows: $94^{\circ} \mathrm{C}$ for $3 \mathrm{~min} ; 40$ cycles of $94^{\circ} \mathrm{C}$ for $30 \mathrm{~s}, 60^{\circ} \mathrm{C}$ for $30 \mathrm{~s}$, and $72^{\circ} \mathrm{C}$ for $1 \mathrm{~min}$; and a final extension at $72^{\circ} \mathrm{C}$ for $10 \mathrm{~min}$.

The bacterial colony with the highest positive value was plated and cultivated overnight. At least 10 positive clones in each test material sample were selected and tested with the Takara incision enzyme by identification of the double-enzyme cut. Positive clones were screened and sent to Shanghai Bioengineering Co., Ltd. for bi-directional sequencing analysis.

\section{RESULTS}

Genome electrophoresis results of the 10 material samples are shown in Figure 1. Amplification results of different trnL target genes and identification of the double-enzyme cut after cloning are presented in Figure 2. Sequencing and positioning/ranking analyses are shown in Table 1.

Lengths of all trnL sequences were within 895 to 935 bp (Table 2). A total of 24 variation sites were detected among the 10 material samples. The basic group variation types 
included transformations of A-G, T-C, and C-T, and transversion of C-A, T-G, G-T, C-G, $\mathrm{A}-\mathrm{T}$, and A-C. Deficiency variations were observed in three regions. Insertion variations with simple reduplications of GCAATA and TTTTTC were also observed in different varieties.

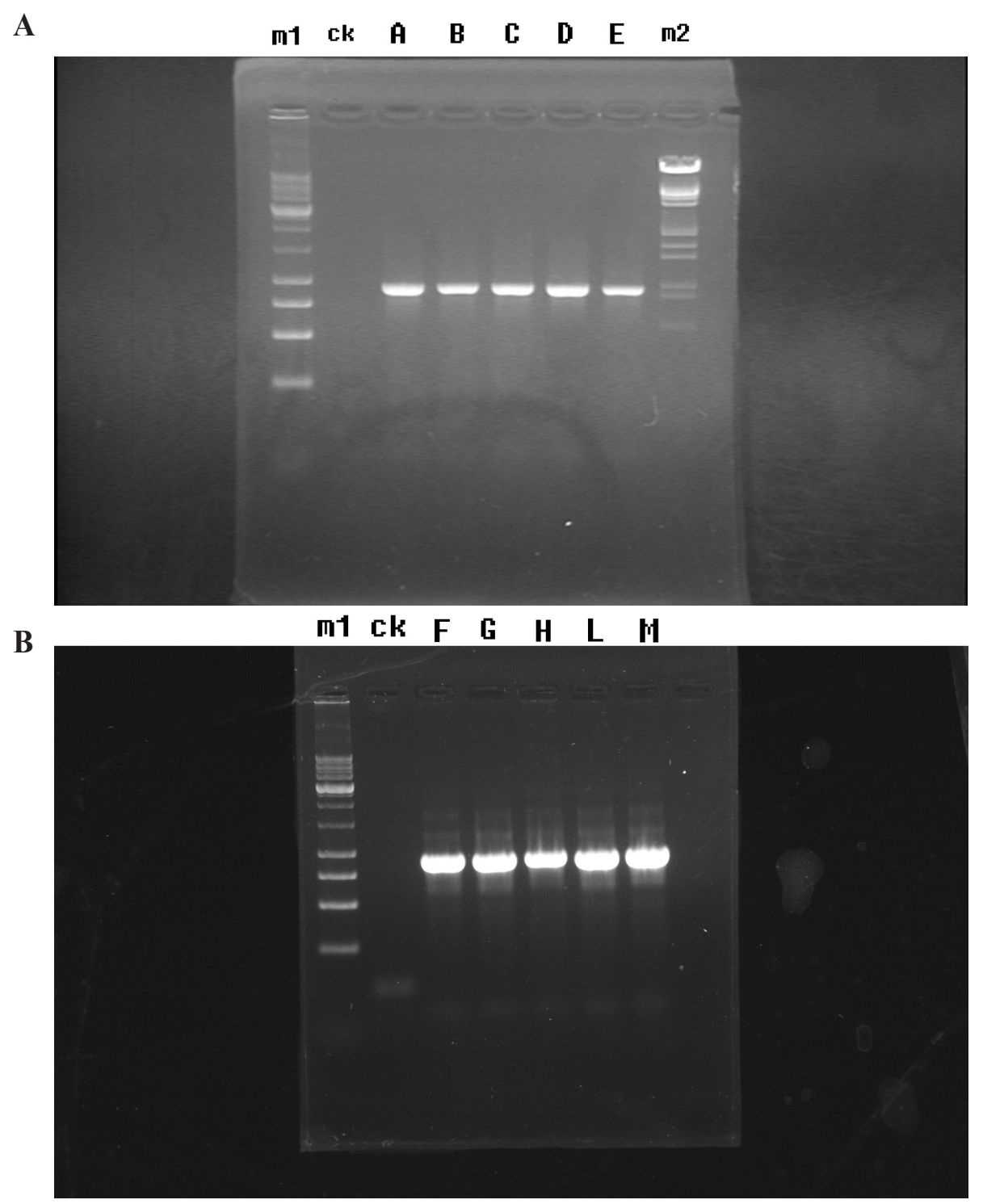

Figure 1. $\operatorname{trnL}$ gene amplification of the 10 Citrus species. A. Lane $m 1=1-\mathrm{kb}$ marker; lane $c k=$ PCR-negative control; lanes $A, B, C, D, E$, and $m 2=\lambda \mathrm{DNA} / E c o \mathrm{RI}+H$ indIII. B. Lane $m 1=1-\mathrm{kb}$ marker; lane ck= PCR-negative control; lanes $F, G, H, L$, and $M$. Lane $A=$ Citrus nobilis Lour. 'Gonggan'; lane $B=C$. haniana Hort. ex Tseng 'Sihuihanggan'; lane $C=C$. flamea Hort. ex Tseng 'Shiyueju'; lane $D=C$. sinensis Osbeck 'Lanhuacheng'; lane $E$ = Atalantia buxifolia (Poir.) Oliv.; lane $F=C$. grandis (L.) Osbeck 'Guanximiyou'; lane $G=$ Poncirus trifoliata (L.) Raf; lane $H=C$. limon (L.) Burm. f. 'Youlikeningmeng'; lane $L=C$. sinensis Osbeck 'Niuheerqicheng'; lane $M=C$. nobilis Lour. 'Xingjinwenzhoumigan'. 
A

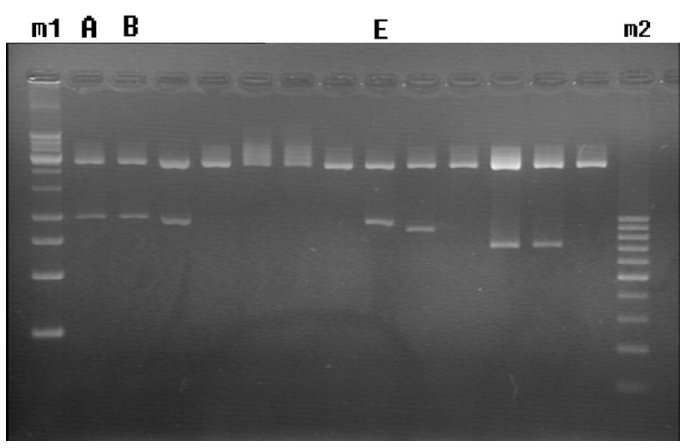

C

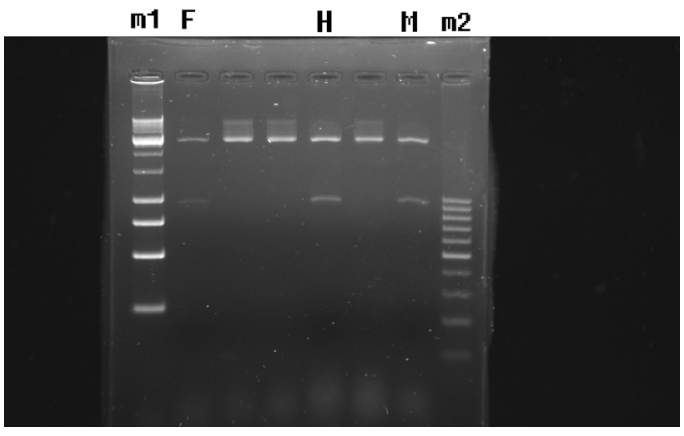

$\mathbf{E}$

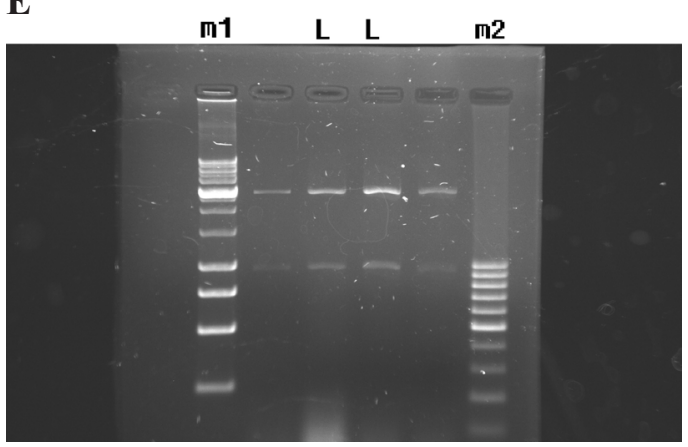

B

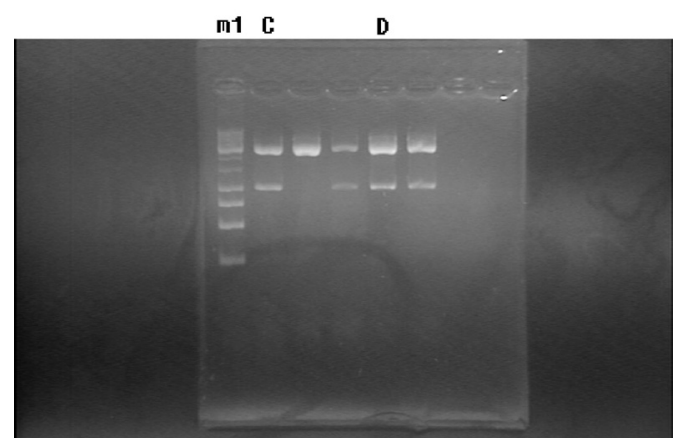

D
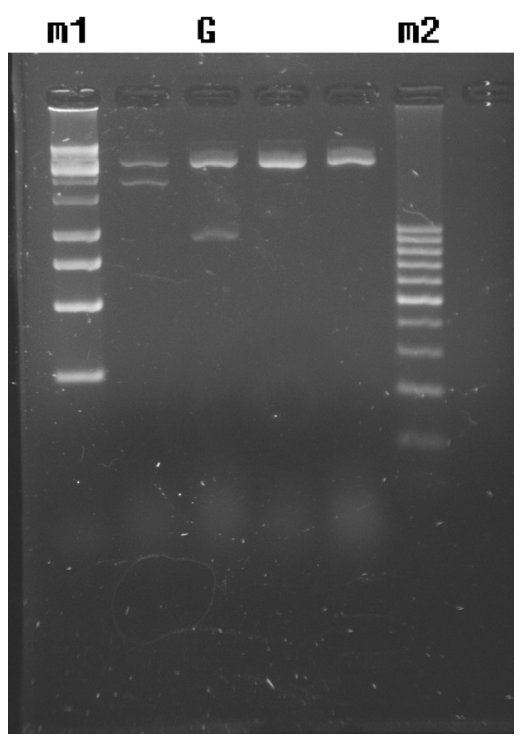

Figure 2. Double-enzyme cut electrophoresis after cloning trnL sequences of the 10 Citrus species. Lane $m 1=$ 1-kb marker; lane $m 2=100$-bp marker; lanes $A$ to $M=$ successful sample about construction of cloning vector and sequencing

The dendrogram (Figure 3) reveals that C. nobilis Lour. 'Gonggan' and C. haniana Hort. ex Tseng 'Sihuihanggan' can be classified in the same category. This category also includes $C$. flamea Hort. ex Tseng 'Shiyueju' and C. nobilis Lour. 'Xingjinwenzhoumigan'. The genetic relationships between other species and the results of their typical morphological classifications showed some similarities. Results of this study are similar to the random amplified polymorphic DNA (RAPD) results previously reported. 
Table 1. Variance analysis of trnL sequences of the 10 Citrus species.

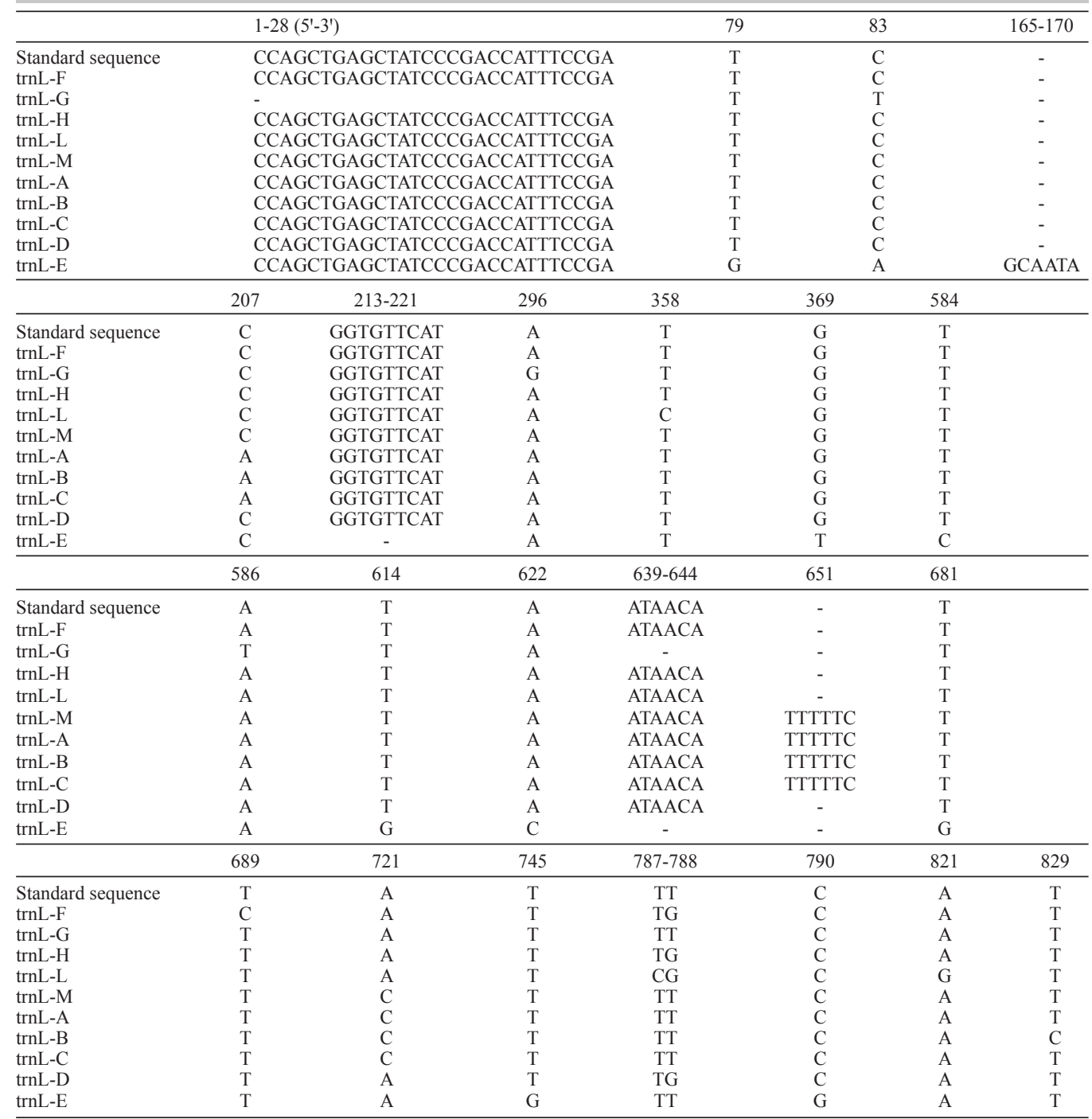

$\mathrm{A}=$ Citrus nobilis Lour. 'Gonggan'; $\mathrm{B}=$ C. haniana Hort. ex Tseng 'Sihuihanggan'; $\mathrm{C}=C$. flamea Hort. ex Tseng 'Shiyueju'; $\mathrm{D}=$ C. sinensis Osbeck 'Lanhuacheng'; $\mathrm{E}=$ Atalantia buxifolia (Poir.) Oliv.; $\mathrm{F}=$ C. grandis (L.) Osbeck 'Guanximiyou'; $\mathrm{G}=$ Poncirus trifoliata (L.) Raf; $\mathrm{H}=$ C. limon (L.) Burm. f. 'Youlikeningmeng'; $\mathrm{L}=C$. sinensis Osbeck 'Niuheerqicheng'; $\mathrm{M}=$ C. nobilis Lour. 'Xingjinwenzhoumigan'.

Table 2. trnL basic group contents of the 10 Citrus species.

\begin{tabular}{ccccccccccc}
\hline & trnL-F & trnL-G & trnL-H & trnL-L & trnL-M & trnL-A & trnL-B & trnL-C & trnL-D & trnL-E \\
\hline Base number & 929 & 895 & 929 & 929 & 935 & 935 & 935 & 935 & 929 & 920 \\
\hline
\end{tabular}

$\mathrm{A}-\mathrm{M}=$ varieties as defined in legend to Table 1 . 


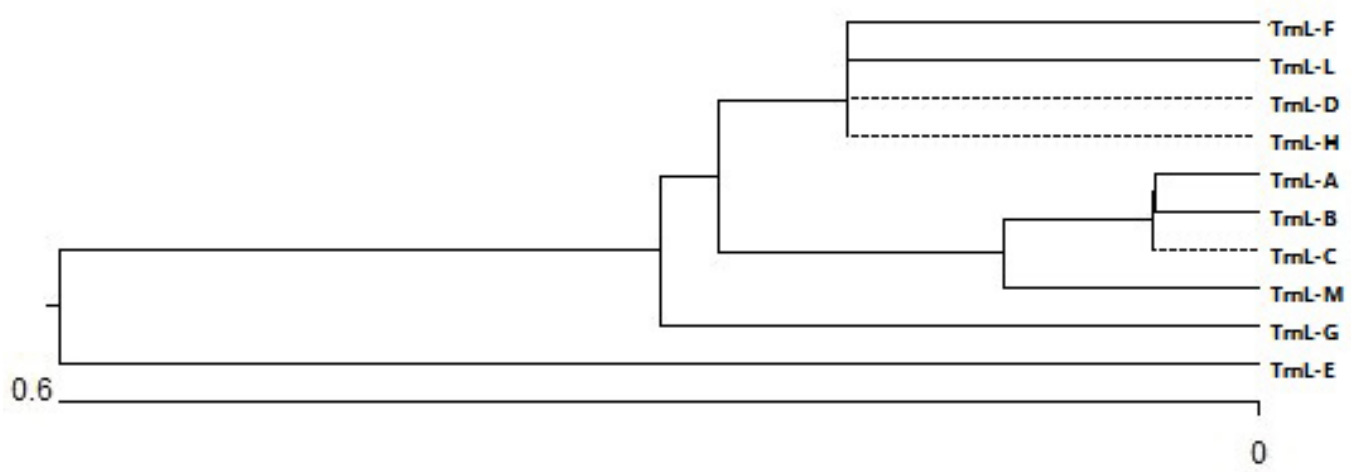

Nucleotide Substitutions (X100)

Figure 3. Dendrogram for the 10 Citrus species derived from cluster analysis of trnL sequences. A-M = varieties as defined in legend to Figure 1.

\section{DISCUSSION}

In recent years, Citrus species have been commonly identified using RAPD, simple sequence repeats, amplified fragment length polymorphism, and similar molecular marker methods. Many studies on the genetic relationships and hybrid origins of Citrus species have also been carried out. These studies have provided useful information for the classification and identification of Citrus. However, information about the chloroplast genomes of Citrus remains limited.

The trnL gene is a gene for encoding leucine tRNA in the chloroplast genomes. Its application in studies of phylogeny and inter-species/species identification has been proven to be effective in short-tube lycoris (Taberlet et al., 1991; Li and Lu, 2005; Yuan et al., 2008). In the present study, we selected lemon, pomelo, Mandarin orange, tangerine, orange, Poncirus, and Atalantia as materials for comparison to construct a phylogeny tree. Clustering results showed that $C$. nobilis Lour. 'Gonggan', $C$ haniana Hort. ex Tseng 'Sihuihanggan', and $C$. flamea Hort. ex Tseng 'Shiyueju' belong to the same category. This category also included C. nobilis Lour. 'Xingjinwenzhoumigan'. A. buxifolia (Poir.) Oliv. and P. trifoliata (L.) Raf belonged to different categories.

The classifications of most species showed that the trnL molecule systematically possesses similarities with typical morphological classification methods. Results are also similar to the dendrogram of Citrus classification acquired by the current authors using RAPD molecular marker methods (Ji et al., 2011). Differences in trnLs generated through systematic evolution can be identified, and these differences represent variations between Citrus species. Thus, the variation sites of trnL sequences can be used in the genealogical classification and species identification of Citrus.

According to farmers from the Sihui County, C. haniana Hort. ex Tseng 'Sihuihanggan' is the biological parent of $C$. nobilis Lour. 'Gonggan', which has a wide circulation. $C$. haniana Hort. ex Tseng 'Sihuihanggan' was already a cultivar before C. nobilis Lour. 'Gonggan' and C flamea Hort. ex Tseng 'Shiyueju' became major varieties in the ZhaoQing area. In addition, C. haniana Hort. ex Tseng 'Sihuihanggan' is nearly extinct (Ji et al., 2010). Signif- 
icant efforts have been made to collect and preserve $C$. haniana Hort. ex Tseng 'Sihuihanggan'. Different citrus varieties were considered the main cultivar during different historical periods in the ZhaoQing area. Today, Citrus cultivars in the ZhaoQing area are fresh cultivars, and are rarely stored and processed. C. haniana Hort. ex Tseng 'Sihuihanggan' ripens every December, requiring time to sweeten after the Spring Festival. Therefore, it is not suitable for the current Citrus industry in the local area and is gradually being eliminated from the market.

The trnL sequence comparison between $C$. nobilis Lour. 'Gonggan' and C. haniana Hort. ex Tseng 'Sihuihanggan' reveals that their sequences are mostly identical except for a variation at $829 \mathrm{bp}$, in which a T/C base transition occurs. This site can be used to differentiate C. nobilis Lour. 'Gonggan' from C. haniana Hort. ex Tseng 'Sihuihanggan'. The chloroplast genome follows maternal inheritance. Therefore, C. nobilis Lour. 'Gonggan' could be a hybrid with $C$. haniana Hort. ex Tseng 'Sihuihanggan' as its female parent. $C$. haniana Hort. ex Tseng 'Sihuihanggan' is of greater importance compared to C. nobilis Lour. 'Gonggan' in terms of theoretical and practical significance in breeding based on storage traits, flavor, and quality changes after storage. The species can serve as a storable variety for breeding and postharvest physiological studies. C. nobilis Lour. 'Gonggan' may also be an appropriate test material for pathological studies on Citrus anthracnose as a sensitive anthracnose variety.

The standard trnL sequences of C. nobilis Lour. 'Gonggan', C. haniana Hort. ex Tseng 'Sihuihanggan', C. flamea Hort. ex Tseng 'Shiyueju', and C. nobilis Lour. 'Xingjinwenzhoumigan' as Sinocitrus tseng are interposed by a short TTTTTC repeated sequence at the 651-bp site. This repeated sequence is not found in the standard trnL sequences of other tested varieties, indicating that this site can be considered a key site in distinguishing Sinocitrus tseng from other species. C. nobilis Lour. 'Gonggan' and C. flamea Hort. ex Tseng 'Shiyueju' have different morphologies, but their trnL sequences reveal that they have a close genetic relationship. C. nobilis Lour. 'Gonggan', C. haniana Hort. ex Tseng 'Sihuihanggan', and $C$. flamea Hort. ex Tseng 'Shiyueju' are the local cultivars of the ZhaoQing area during different historical stages. They all have the same trnL sequences of $207 \mathrm{bp}$, a feature that other varieties do not possess. C-A transversion occurs in these three varieties compared to other Citrus varieties.

\section{CONCLUSIONS}

C. nobilis Lour. 'Gonggan', C. haniana Hort. ex Tseng 'Sihuihanggan', and C. flamea Hort. ex Tseng 'Shiyueju' are the local cultivars of the ZhaoQing area during different historical stages and have close genetic relationships. C. nobilis Lour. 'Gonggan' is probably a hybrid with $C$. haniana Hort. ex Tseng 'Sihuihanggan' as its female parent.

Differences in trnL genes caused by systematic evolution allow the determination of variations between Citrus varieties. Variation sites of trnL sequences can be used in the phylogenetic classification and species identification of Citrus.

\section{ACKNOWLEDGMENTS}

Research supported by the Science and Technology Planning Project of Guangdong Province (\#2010B080201006) and the Science and Technology Planning Project of Zhaoqing City (\#2009N029). 


\section{REFERENCES}

Green RM, Vardi A and Galun E (1986). The plastome of Citrus: physical map, variation among Citrus cultivars and species and comparison with related genera. Theor. Appl. Genet. 72: 170-177.

Ji QH and Guo YJ (2011). China Gonggan (Citrus nobilis Lour. 'Gonggan'). Science Press, Beijing.

Ji QH, Guo YJ, Yao JM and He JH (2010). Study on leaf mineral nutrient and its effect on fruit quality of Gonggan (Citrus reticulata Blanco var. gonggan). South. China J. Agric. Sci. 23: 786-790.

Ji QH, Zeng JW and Guo YJ (2011). Using optimized random amplified polymorphic DNA (RAPD) markers to identify the category status of Citrus nobilis Lour. Gonggan. Afr. J. Biotechnol. 10: 13982-13990.

Li CX and Lu SG (2005). Relationship of Microsorium membranaceum var. carinatum and Microsorium membranaceum var. membranaceum based on the sequence analysis of chloroplast rbcL, rps4, $\operatorname{trnL}-\operatorname{trnF}$ and $\operatorname{rps} 4$-trnS. Biodivers. Sci. 13: 174-179.

Taberlet P, Gielly L, Pautou G and Bouvet J (1991). Universal primers for amplification of three non-coding regions of chloroplast DNA. Plant Mol. Biol. 17: 1105-1109.

Wang FS and Jiang D (2010). Studies on genetic background of important germplasm resources among Citrus based on cpSSR and EST-SSR marker. Acta Horticult. Sin. 37: 465-474.

Xie RJ, Zhou ZQ and Deng L (2008). Taxonomic and phylogenetic relationships among the genera of The True Citrus Fruit Trees Group (Aurantioideae, Rutalea) based on AFLP Markers. J. Syst. Evol. 46: 628-691.

Yuan JH, Sun S, Peng F, Feng X, et al. (2008). Genetic variations in trnL-F sequence and phylogenetic clustering of Lycoris species. Zhongguo Zhong Yao Za Zhi 33: 1523-1527. 